\title{
Magnetocaloric Properties of Severe Plastic Deformed $\mathrm{Gd}_{100-x} \mathrm{Y}_{x}$ Alloys
}

\author{
S. Taskaev ${ }^{a, b, c}, \mathrm{~K} . \mathrm{Skokov}^{d}$, D. Karpenkov ${ }^{d}$, V. Khovaylo ${ }^{b, e}, \mathrm{~V}$. Buchelnikov ${ }^{a}$, \\ D. Zherebtsov ${ }^{c}$, M. Ulyanov $^{a, *}$, D. Bataev ${ }^{a}$, M. DrobosyuK $^{a}$, A. Pellenen $^{c}$ \\ ${ }^{a}$ Faculty of Physics, Department of Physics, Chelyabinsk State University, 454001 Chelyabinsk, Russia \\ ${ }^{b}$ National University of Science and Technology "MISIS", 119049 Moscow, Russia \\ ${ }^{c}$ National Research South Ural State University, 454080 Chelyabinsk, Russia \\ ${ }^{d}$ Department of Physics, Tver State University, 170100 Tver, Russia \\ e ITMO University, 197101 St. Petersburg, Russia
}

\begin{abstract}
In this work we report on the results of investigation of the magnetocaloric effect in $\operatorname{Gd}_{100-x} \mathrm{Y}_{x}(x=0,5$, $10,15)$ cold rolled ribbons. It is shown that the magnetocaloric effect exists within a wide temperature interval 258-295 K and is comparable with magnetocaloric effect observed in bulk samples of pure gadolinium. The value of the magnetocaloric effect in the rolled samples is reduced as compared with the bulk samples and strongly depends on the degree of the plastic deformation. Heat treatment procedures can restore the values of the magnetocaloric effect in the cold rolled ribbons up to initial ones. Thus, cold rolling is proposed to be a promising technique for producing thin forms of magnetocaloric materials for heat exchangers of magnetic cooling devices, modifying microstructure of the materials and related magnetic properties.
\end{abstract}

DOI: 10.12693 /APhysPolA.127.641

PACS: $75.30 . \mathrm{Sg}, 75.30 . \mathrm{Gw}, 75.50 . \mathrm{Cc}$

\section{Introduction}

Gadolinium is a benchmark material in the magnetic refrigeration technology. As a rule, all magnetic and thermodynamical properties of magnetocaloric (MCE) materials are compared with the properties of pure Gd. Since the discovery in 1997 of the "giant magnetocaloric effect" [1] the development of magnetic refrigeration has been growing exponentially. Since that event numerous different families of alloys have been found and investigated, see for instance [2]. Some of them are used in known prototypes of magnetic refrigerators and heat pumps, a review of the machines built before the year 2010 can be found in [3]. Nevertheless, up to now Gd and Gd-based alloys with first or second order transitions are still one of the best materials for prototyping magnetic refrigerators.

The aim of this work is to investigate the magnetic and magnetocaloric properties of the series of intermetallic alloys $\mathrm{Gd}_{100-x} \mathrm{Y}_{x}(x=0,5,10,15)$ treated with the help of severe plastic deformation (SPD) technique. The principal idea of this work is to obtain information about the influence of SPD on magnetic and magnetocaloric properties of the alloys under investigation. Milstein et al. [4] had reported on cold rolling of Gd polycrystalline ingot, but the thickness reduction in those experiments was amounted only by $27 \%$. Today there is no data on the influence of cold rolling on magnetic or magnetocaloric properties, except Gd [5].

*corresponding author; e-mail: max-39@yandex.com
The solubility of yttrium in gadolinium is quite high and this fact allows us to prepare alloys with wide range of concentrations [6]. From that point of view Gd-Y alloys are very interesting model alloys for investigating the influence of severe plastic deformation on magnetic and magnetocaloric properties of diluted magnetic systems.

SPD technique is very convenient for designing new functional materials. Magnetic, structural or thermodynamic properties of materials can be modified by a degree of plastic deformation and this has not only academic interest, but also is of interest for practical applications. For instance, one of the possible ways for designing materials for magnetic refrigeration is tightly connected with preparation of very thin (down to several micrometers) foils with high MCE and good mechanical properties. But as has been shown in our previous works, SPD leads to a significant depression of the magnetocaloric effect in SPD Gd foils.

The reason of such behavior lies in magnetic anisotropy induced during SPD treatment. So unusual phenomena drive to new thermodynamic and magnetic properties of deformed Gd foils [5] and make its future not so bright for magnetocaloric application. But special hightemperature heat treatment can restore magnetocaloric properties of the cold rolled ribbons back to the bulk ones. The heat treatment regimes are directly connected with the degree of deformation.

\section{Samples preparation}

The starting materials were $99.9 \%$-pure gadolinium and yttrium metals. Four ingots of $\mathrm{Gd}_{100-x} \mathrm{Y}_{x}(x=0$, $5,10,15)$ were prepared by arc melting in argon atmo- 
sphere. X-ray analysis showed that all samples are single phase.

Cold rolling procedure was performed with the help of four roller mill. The work rollers were made from tungsten carbide and have the diameter of $40 \mathrm{~mm}$. Speed of the rolling was $0.05 \mathrm{~m} / \mathrm{s}$. The maximal stress produced by mill was $50 \mathrm{kN}$. All specimens were treated by cold rolling procedure with many cycles of plastic deformation. On each cycle an additional deformation increased slowly to avoid heating the material.

Some characteristics of as-cast ingots and rolled materials are shown in Table. Width of the as-cast alloy means the height of the alloy ingot; length means the maximal diameter of the ingot. Deformations were determined as a relation between the widths of a sample before rolling and after the last roling cycle.

Optical image of the rolled samples and scanning electron microscopy of transverse fracture of cold rolled ribbons are shown in Figs. 1 and 2, respectively. Ribbons of the rolled alloys are flexible despite numerous cracks. The structure of the ribbon is well texturized in the plane of cold rolling (see Fig. 2).

TABLE

Residual deformation of the rolled alloys.

\begin{tabular}{c|c|c|c}
\hline \hline Composition & $\begin{array}{l}\text { Initial size } \\
\text { of the ingot } \\
\text { (width/length) } \\
{[\mathrm{mm}]}\end{array}$ & $\begin{array}{c}\text { Width of } \\
\text { rolled foil } \\
{[\mathrm{mm}]}\end{array}$ & $\begin{array}{c}\text { Deformation } \\
w_{\text {start }} / w_{\text {finish }}\end{array}$ \\
\hline $\mathrm{Gd}$ & $6.0 / 16.0$ & $0.04-0.10$ & $60-150$ \\
$\mathrm{Gd}_{95} \mathrm{Y}_{5}$ & $6.3 / 16.0$ & $0.10-0.12$ & $53-63$ \\
$\mathrm{Gd}_{90} \mathrm{Y}_{10}$ & $6.5 / 16.5$ & $0.10-0.12$ & $54-65$ \\
$\mathrm{Gd}_{85} \mathrm{Y}_{15}$ & $6.25 / 17.5$ & $0.10-0.12$ & $52-63$
\end{tabular}

\section{Results and discussion}

Direct measurements of adiabatic temperature change $\Delta T_{a d}$ were carried out in an apparatus described in detail elsewhere [7]. The variable magnetic field was generated by a permanent-magnet assembly and was limited to 1.9 $\mathrm{T}$. The field was applied in the plane of the rolled strips, to avoid demagnetization. The acquired $\Delta T_{a d}$ data for bulk samples and rolled samples are plotted against temperature in Fig. 3. The graph also contains a reference curve measured on polycrystalline Gd. One can appreciate that cold rolling depresses the $\Delta T_{a d}$ effect in $\mathrm{Gd}$ to about one-half of the initial value and this depression is connected with the degree of plastic deformation (see Table).

Two different tendencies in MCE of studied samples can be noticed (see Fig. 4). For the case of bulk alloys a depression of the magnetocaloric effect during increasing yttrium content in the sold solution occur, but in the case of deformed samples this tendency brakes and we see increasing magnetocaloric effect. This discrepancy can be described by the fact that occasionly we measured the

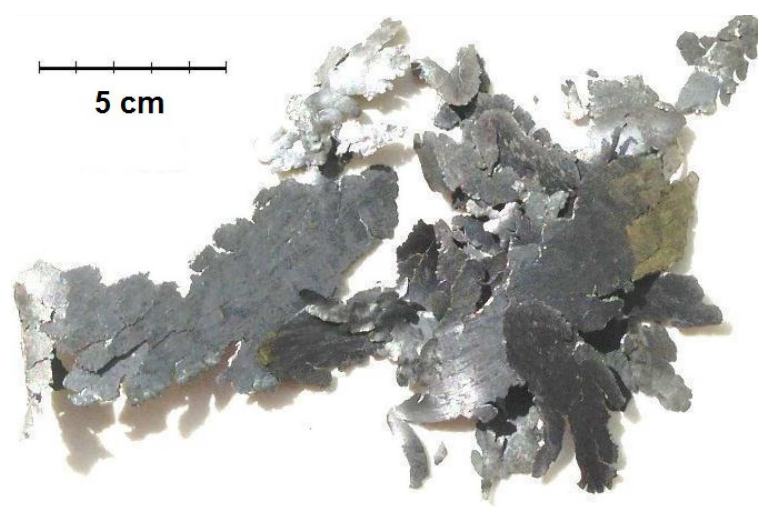

Fig. 1. Optical image of rolled samples of Gd-Y alloys.

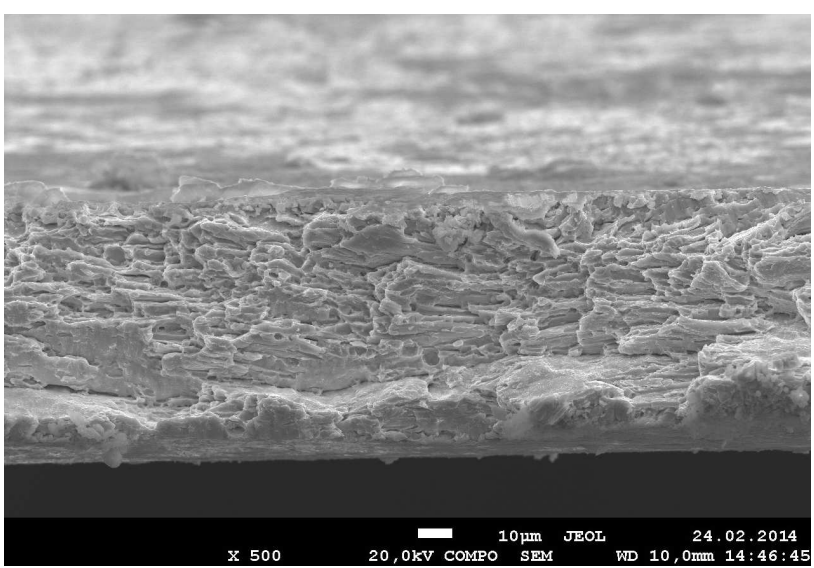

Fig. 2. EM image of transverse fracture of rolled sample.

most deformed sample of pure gadolinium and the magnetocaloric effect was much lower. If that the first point which correspond to cold rolled alloy with zero yttrium content should be higher and both dependences sould correlate.

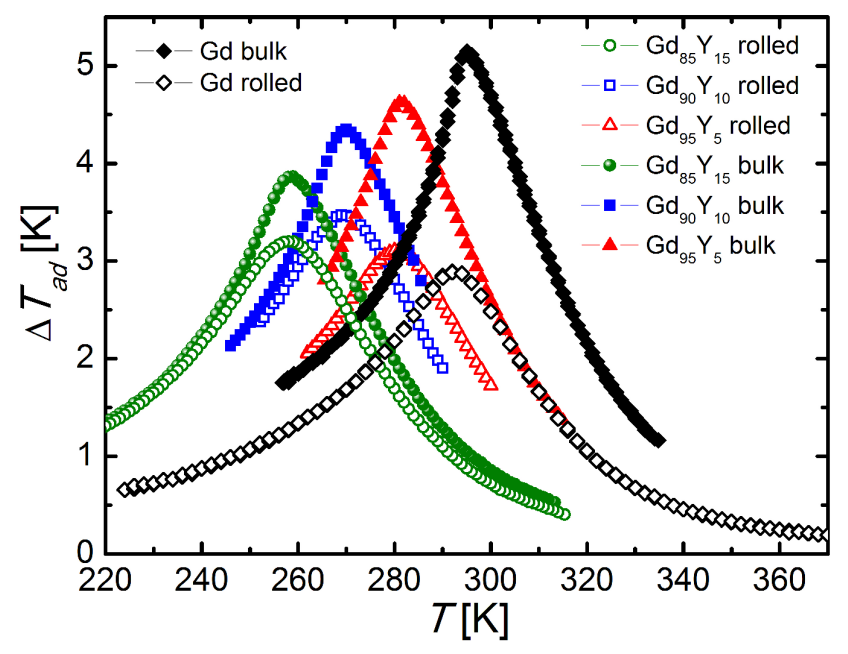

Fig. 3. Magnetocaloric effect in bulk and rolled samples of $\mathrm{Gd}_{100-x} \mathrm{Y}_{x}(x=0,5,10,15)$. 
Curie temperature $T_{\mathrm{C}}$ as a function of yttrium contents for bulk and rolled samples of $\mathrm{Gd}_{100-x} \mathrm{Y}_{x}$ is shown in Fig. 5. Good coincident for both series of samples been observed. $T_{\mathrm{C}}$ linearly decreases with increasing yttrium concentration within the interval 258-295 K. The same trend has been observed in previous studies [6]. Difference between [6] and present work in the region of high yttrium contents is probably due to a deviation from nominal compositions. The decrease of $T_{\mathrm{C}}$ is observed due to a reduction of the exchange interactions with increasing nonmagnetic $\mathrm{Y}$ atoms concentration. It has to be noticed that for $\operatorname{Gd}_{100-x} \mathrm{Y}_{x}(x=0,5,10,15)$ alloys the temperature span achieved about $37 \mathrm{~K}$ and this is quite large useful interval for different magnetocaloric applications and devices.

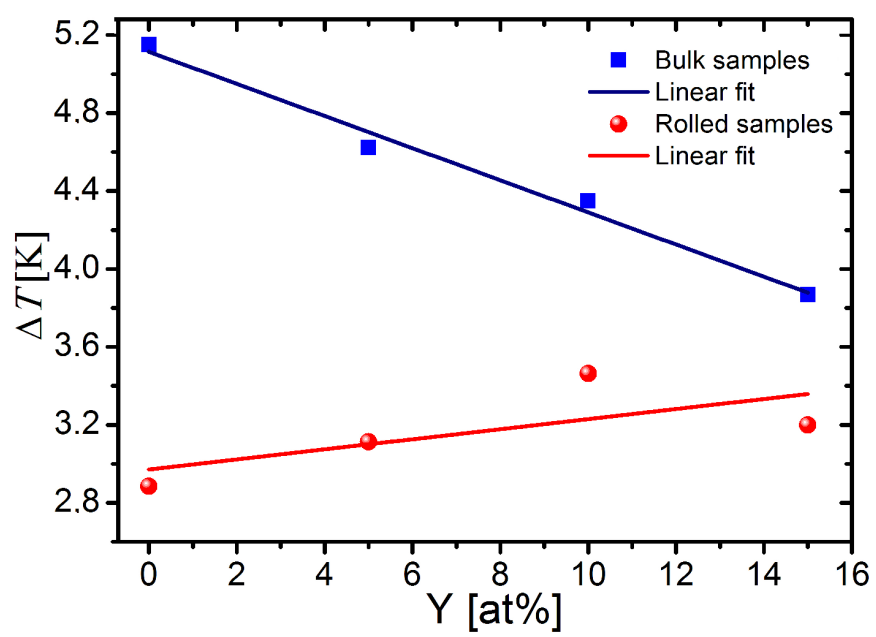

Fig. 4. Phase diagram of magnetocaloric effect in bulk and rolled samples of $\operatorname{Gd}_{100-x} \mathrm{Y}_{x}(x=0,5,10,15)$.

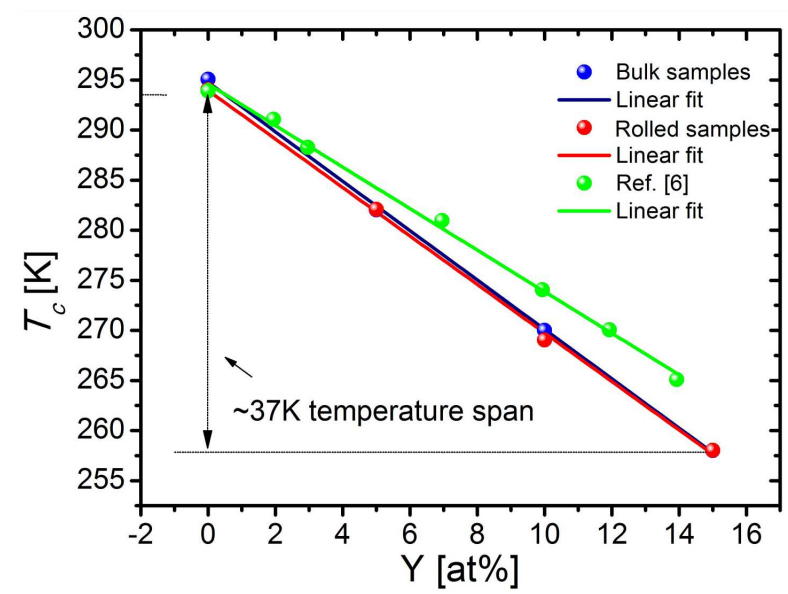

Fig. 5. Phase diagram of Curie point in bulk and rolled samples of $\operatorname{Gd}_{100-x} \mathrm{Y}_{x}(x=0,5,10,15)$.

\section{Conclusions}

Cold rolling as a severe plastic deformation method is quite suitable technique for producing thin ribbons of some functional materials. It is completely inapplicable for brittle intermetallic alloys or some pure elements like cobalt, because even small deformations applied by rollers results in destruction of the sample. Nevertheless in the case of gadolinium-yttrium alloys this technique allows us to produce ribbons with a thickness down to 40 micrometers which can be used in magnetocaloric heat exchangers. The width of the magnetocaloric materials is principal in heat exchangers, because the process of heat transfer is limited mainly by the heat conduction. In this case the larger is the surface to volume ratio, the better system will transfer heat. The theoretical background of this was discussed in [8].

Studied $\operatorname{Gd}_{100-x} \mathrm{Y}_{x}(x=0,5,10,15)$ alloys show perfect temperature span of $T_{\mathrm{C}}$ (about $37 \mathrm{~K}$ ) and good mechanical properties for subsequent treatment. No changes were observed for Curie temperatures for appropriate compounds. During SPD treatment of $\mathrm{Gd}_{100-x} \mathrm{Y}_{x}$ $(x=0,5,10,15)$ we observe a depression of magnetocaloric effect by $20-50 \%$ as compared to the bulk samples. The origin of this lies in the magnetic anisotropy induced during cold rolling. For the considered values of deformations it is quite simple to recover magnetocaloric effect up to bulk value by high-temperature heat treatment [9].

\section{Acknowledgement}

The authors gratefully acknowledge the financial support of the Ministry of Education and Science of the Russian Federation in the framework of Increase Competitiveness Program of MISiS and financial support of the RFBR 12-07-00676-a and RF President MD-770.2014.2 grants.

\section{References}

[1] K.A. Gschneidner, Jr., V.K. Pecharsky, Int. J. Refrig. 31, 945 (2008).

[2] K.A. Gschneidner, Jr., V.K. Pecharsky, A.O. Tsokol, Rep. Prog. Phys. 68, 1479 (2005).

[3] B. Yu, M. Liu, P.W. Egolf, A. Kitanovski, Int. J. Refrig. 33, 1029 (2010).

[4] F. Milstein, J.A. Baldwin, Jr., T.W. James. J. Appl. Phys. 44, 4824 (1973).

[5] S.V. Taskaev, M.D. Kuz'min, K.P. Skokov, D.Yu. Karpenkov, A.P. Pellenen, V.D. Buchelnikov, O. Gutfleisch, J. Magn. Magn. Mater. 331, 33 (2013).

[6] S. Kaji, T. Kobayashi, A.T. Saito, J. Phys.: Conference Series 200, 032029 (2010).

[7] K.P. Skokov, V.V. Khovaylo, K.-H. Muller, J.D. Moore, J. Liu, O. Gutfleisch, J. Appl. Phys. 111, 07A910 (2012).

[8] M.D. Kuz'min, Appl. Phys. Lett. 90, 251916 (2007).

[9] S.V. Taskaev, V.D. Buchelnikov, A.P. Pellenen, M.D. Kuz'min, K.P. Skokov, D.Yu. Karpenkov, D.S. Bataev, O. Gutfleisch, J. Appl. Phys. 113, 17A933 (2013). 